This item was submitted to Loughborough's Research Repository by the author.

Items in Figshare are protected by copyright, with all rights reserved, unless otherwise indicated.

\title{
Approaches and challenges for the manufacture and scale-out of autologous cell therapies
}

PLEASE CITE THE PUBLISHED VERSION

https://doi.org/10.18609/cgti.2017.076

PUBLISHER

Bioinsights

VERSION

VoR (Version of Record)

\section{PUBLISHER STATEMENT}

This work is made available according to the conditions of the Creative Commons Attribution-NonCommercialNoDerivatives 4.0 International (CC BY-NC-ND 4.0) licence. Full details of this licence are available at: https://creativecommons.org/licenses/by-nc-nd/4.0/

\section{LICENCE}

CC BY-NC-ND 4.0

\section{REPOSITORY RECORD}

Harrison, Richard P.. 2019. "Approaches and Challenges for the Manufacture and Scale-out of Autologous Cell Therapies”. figshare. https://hdl.handle.net/2134/27870. 


\section{CELL \& GENE THERAPY INSIGHTS}

STRATEGIES FOR SCALE-UP

\& SCALE-OUT

\section{SPOTLIGHT}

\section{Approaches to the manufacture and scale-out of autologous cell} therapies

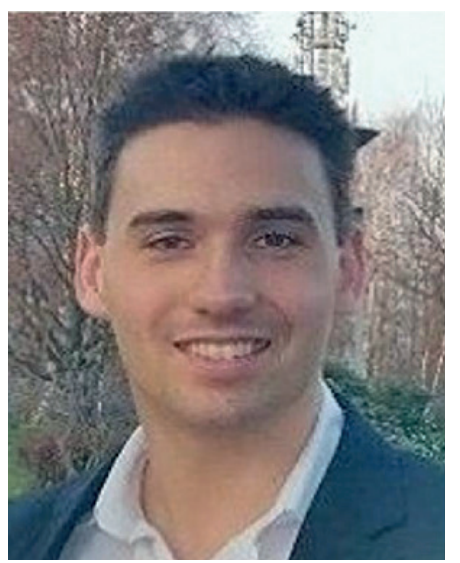

Dr Richard Harrison is an EPSRC ETERM Fellow at Loughborough University. His research is primarily focused on the manufacturing of advanced therapies, more specifically the future manufacturing paradigms these advanced therapies will require. As these advanced healthcare products do not fit easily into existing manufacturing solutions, Richard's research examines the business models, distribution networks, cost of goods and reimbursement strategies that may suit these therapies, and cost of goods modeling. A particular focus of this strategy is investigating the feasibility of decentralized manufacturing of advanced therapies across diverse geographical regions. Richard holds a BSc in Biochemistry, an MSc in Stem Cell Technologies and a PhD in targeting of cell therapies. He was awarded his E-TERM Landscape Fellowship in 2016 and is currently based at Loughborough University with Visiting Fellow status at The University of Nottingham.

With all the positive news surrounding the fantastic recent milestone of two autologous CAR-T therapies approved by the FDA, as an outsider looking in you could be forgiven for assuming that the manufacture of these therapies is a straightforward process. Do you feel this is an accurate representation?

\section{T} vancing steadily. We are learning a lot from them and still have a way to go, and I hope they'll give us the strength we need to push forward in the wider cell therapy field. 
As I see it, manufacturing of these therapies is not simple and there are some key issues that need addressing in particular for CAR-T manufacturing and this connects back to the cost of goods.

The biology of CAR-T therapies is not perfectly understood and this makes developing consistent process from highly variable starting biological materials extremely challenging. In addition, if you're going to scale-out your process massively, you really need to automate as much as possible. The lack of hardware for online testing of function or potency makes designing robust automatable platforms very challenging.

Similarly, we're lacking effective standards to benchmark what we're doing and therefore the systems and products can suffer from the drift. Finally, we're suffering from logistics and formulation issues. Cryopreservation is a great solution because you can store your product and it is ideal for shipping. But if you lose a significant portion of your product during this process, your product may not be quite as you expect when it arrives to the patient and the efficacy could be compromised.

Therefore, if you're going down a heavily scaled out route, the overall costs of goods will be high, and we have already started seeing it in the headline price tags for the released autologous products. There have already been accusations of price gouging, and I don't think that's very healthy for the field. It would certainly be beneficial if the prices could come down following progress in the points mentioned.

\section{Q What are the unique challenges faced by manufacturers of more-than-minimally manipulated autologous cell-based therapies in developing scale- out manufacturing processes?}

The challenges faced by scale-up processes. Logistics is a key component of scale-out manufacturing and the real challenge associated with scale-out is its complex logistical burden.

Although utilizing decentralized manufacturing to manufacture these products presents an attractive solution for implementing the roll out of patient-specific cell and gene therapies, maintaining multiple manufacturing sites becomes a challenge all on its own. We've looked at heavily scaled out facilities on the $\mathrm{WAVE}^{\ominus}$ reactor producing two therapeutic doses by reactor, and the space you need alone for that is very large. And the starting requirements of maintaining a large number of small vessels are significantly higher than a more scalable platform with multiple doses per vessel.

Another issue we've been grappling with from decentralized manufacturing is if you're distributing manufacturing across geographic regions, maintaining comparability becomes a real challenge. If you have your analytical equipment that you use to demonstrate your comparability with the assays you develop and calibrate to standards, the pieces of equipment alone can vary significantly, and then the standards can vary again due to age, transport, operator experience and the cumulative variation adds up and you end up drifting from specification. 
Addressing logistical concern is something we're just beginning to catch up with. There have been problems in the past trying to connect various links in the supply chain. If all the factors - delivery, manufacturing input or output depending on if you're collecting donor material and shipping to another site, surgeon readiness, patient readiness, or if you don't have a product you can store or a place to store which is often the case in a clinical facility - don't align correctly, then you've lost your product. And there can be loses as high as 50\%, which bumps up the cost of goods massively. And it really changes the value proposition for your product.

How does variability of the starting material impact scale-out processes and what are the control strategies in place to achieve manufacturing consistency?

ariability of input or starting materials is a critical factor influencing the prospects for reproducible manufacturing. Differences between tissue sources and individual donor characteristics, in addition to the poor capability of isolation methods create greater potential for product variability in an autologous setting.

We can put simple control strategies in place to help achieve consistency. Introducing automation is a great way to minimize human error and risk of contamination. However, we need flexibility in the process, pushing these too far, for instance, trying to automate the process too heavily will result in losing control over the process. A better understanding of the input variables would allow the quality control of the source and nature of starting materials in the future.

\section{Q}

Aside from manufacturing platforms, what other strategies are being developed in attempts to reduce the cost of goods of autologous products?

\footnotetext{
Aside from manufacturing challenges, two key problems the industry face is that firstly, we can't really characterize the source material before it arrives on site and secondly, we have only few tests available to predict function. Understanding what's going into the process is critical if we're considering a decentralized model or scaleout manufacturing, because patient material is inherently variable. With the dramatic drop of cost of sequencing techniques and the reduction in operating complexity, I think we will reach a point where we obtain that information in almost real-time, or as it is en route to manufacturing sites. Through this we can basically triage our donor material into batches, and predicate how it will behave before it actually begins to in the manufacturing process. Thus, we should be able to respond significantly better than we are currently able to. That comes back to using material a lot more efficiently and reducing cost of goods. One of the most recent technologies around that are cost of good models, designed particularly to predict process costs early on, and help make decisions on which the
} 
winning products are, which are not, and when you can decide to lock down a process as well. If there's high variability in your process, that might have a huge impact on your overall cost of goods and you will need to focus on locking that down and trying to get to grips with that.

There's only a finite amount of money in the field, using it as efficiently as possible is the key.

Q

When each patient's cell production is considered one batch, setting tight specifications for product release to meet a cGMP requirement is difficult. What's the industry doing to overcome this challenge?

There are two aspects to having highly variable material. One is we can't set tight standards now because we've got an inherently variable production process, and it needs that wiggle room for further modifications. The second is developing the methods to practically identify individualized characteristics of the product undergoing the manufacturing process and apply the appropriate specification requirement.

Ultimately it comes back again to the understanding that ours is a young field. To rapidly characterize the input material and extrapolate characteristics to determine the handling requirements all in a short time frame, and at a price point that isn't massively driving up your price per lot is really challenging for single dose per lot therapies.

Q

What are some of the regulatory challenges for the manufactureandscale-outofautologouscelltherapies? How critical is global regulatory harmonization in the manufacture and commercialisation of cell therapies?

A lot of our recent research has been in investigating the feasibility of decentralized or redistributed manufacturing. One of the recurring questions we have is how would decentralized manufacturing address regulatory concerns specifically around maintaining standards and quality at a distance. Probably this could be achieved with a reduced staff number, because you want to have as bare-bones facility as possible to reduce the cost of goods.

Ultimately, I see the solution being driven by technology - having process control modules that can manage your reactors and transport, and monitor everything in your facility and en route to your facility, via a network. There are technologies like TrakCel's ColdTrak that help inform what's going on across your network. I think as these technologies evolve, it will not only be used for monitoring purposes but also for enforcing at a distance. That's going to really help maintain a geographical network and enforce standards remotely.

As the concepts underlying Industrie 4.0 become more common, and the hardware and peripherals become increasingly 'smart', it will become much easier to pull these solutions together to form responsive solutions that should help support regulatory acceptance of decentralized manufacturing. 
Many see regulation as a hindrance and some see it as helpful. In regard to the harmonization, it's only going to be a positive step in simplifying the landscape for advanced therapies. But the caveat is that, it's still a young field, regulators need to remain engaged in understanding the limitations of what we're actually able to know about advanced therapies. Unlike traditional pharmaceuticals, cells are complex entities and are sensitive, and we're only just beginning to understand and control them.

As long as the regulators understand that, which I think the regulatory bodies in EU, USA, Japan and Canada do, this is the correct approach. As long as we can get robust responsive contingencies in our processes, to anticipate variation, we should be moving to a place where we're more able to accept them. production for more common disease indications?

F From my perspective, one of the worrying aspects I have is that the production cost figures for autologous therapies are just too high at the moment. I don't see how we can begin to manufacture therapies at a scale with cost of goods per lot, which can be considered feasible for a blockbuster therapy. If we manage to treat one of the real blockbuster therapies, we're going to place unrealistic demands on our healthcare system for the ability to pay for them.

I see the demand for the current generation of CAR-T therapies really pushing the field forwards. The strong demand by patients and advocacy groups combined with the relatively high efficacy is extremely promising. Consequently, the fact these processes often contain manual processes, and improvised equipment, reagents and processes are able to be somewhat overlooked due to the high demand. In doing so, we are fuelling progress in establishing the next generation of robust, unified manufacturing processes.

\section{AFFILIATION}

Richard P Harrison, EPSRC E-TERM Landscape Fellow, Centre for Biological Engineering, Loughborough University, UK

Wolfson Centre for Stem cells, Tissue Engineering and Modelling (STEM), The University of Nottingham, UK

\footnotetext{
c) $\$($ This work is licensed un-

der a Creative Commons

Attribution - NonCommercial - NoDeriva-

tives 4.0 International Licens
} 